\title{
Centralized versus decentralized control-A solvable stylized model in transportation
}

\author{
Max-Olivier Hongler ${ }^{\mathrm{a}}$, Olivier Gallay ${ }^{\mathrm{a}, *}$, Michael Hülsmann ${ }^{\mathrm{b}}$, Philip Cordes ${ }^{\mathrm{b}}$, \\ Richard Colmorn ${ }^{\mathrm{b}}$ \\ ${ }^{a}$ Ecole Polytechnique Fédérale de Lausanne (EPFL), STI-IMT-LPM, Station 17, CH-1015 Lausanne, Switzerland \\ ${ }^{\mathrm{b} J a c o b s}$ University, Campus Ring 1, DE-28759 Bremen, Germany ${ }^{1}$
}

\section{A R T I C L E I N F O}

\section{Article history:}

Received 29 December 2009

Received in revised form 29 March 2010

Available online 4 June 2010

\section{Keywords:}

Transportation logistics

"Smart parts" dynamics

Multi-agent systems

Mean-field approximation

Burgers' nonlinear evolution

Emergent cooperative pattern

Optimal stochastic control

Kullback-Leibler relative entropy

\begin{abstract}
A B S T R A C T
Today's supply networks consist of a certain amount of logistics objects that are enabled to interact with each other and to decide autonomously upon their next steps; in other words, they exhibit a certain degree of autonomous cooperation. Therefore, modern logistics research regards them as complex adaptive logistics systems. In order to analyze evolving dynamics and underlying implications for the respective systems' behavior as well as the potential outcomes resulting from the interaction between autonomous decisionmaking "smart parts", we propose in this contribution a fully solvable stylized model. We consider a population of homogeneous, autonomous interacting agents traveling on $\mathbb{R}$ with a given velocity that is itself corrupted by White Gaussian Noise. Based on real time observations of the positions of his neighbors, each agent is allowed to adapt his traveling velocity. These agent interactions are restricted to neighboring entities confined in finite spatial clusters (i.e. we have range-limited interactions). In the limit of a large population of neighboring agents, a mean-field dynamics can be derived and, for small interaction range, the resulting dynamics coincides with the exactly solvable Burgers' nonlinear field equation. Explicit Burgers' solution enables to explicitly appreciate the emergent structure due to the local and individual agent interactions. In particular, for strongly interactive regimes in the present model, the resulting spatial distribution of agents converges to a shock wave pattern. To compare performances of centralized versus decentralized organization, we assign cost functions incurred when velocity adaptations are triggered either by multi-agent interactions or by central control. The multi-agent cumulative costs are then compared with the costs that would be incurred by implementing an effective optimal central controller able, for a given time horizon, to reproduce an identical spatial probability distribution of agents. The resulting optimal control problem can be solved exactly and the corresponding costs can be expressed as the Kullback-Leibler relative entropy between the free and the controlled probability measures. This enables one to conclude that for time horizons shorter than a critical value, multi-agent interactions generate smaller cumulative costs than an optimal effective central controller.
\end{abstract}

(c) 2010 Elsevier B.V. All rights reserved.

\section{Introduction-Smart parts transportation logistics}

In the daily life, many of our decisions are taken with limited rationality. Indeed, the lack of complete information, the huge number of control parameters and criteria together with their nonlinear relationship and the ubiquitous presence of random fluctuations corrupting preplanned strategies force us to take actions with relative uncertainty. Highly complex,

\footnotetext{
* Corresponding author.

E-mail address: olivier.gallay_1@a3.epfl.ch (0. Gallay).

1 Collaborative Research Centre 637: Autonomous Co-operating Logistic Processes-A Paradigm Shift and its Limitations.
} 
multicriteria decision issues in modern management raise basically similar difficulties, thus questioning the overall relevance of centralized organization processes.

Logistics is one field of application in which recent developments of information and communication technologies, such as RFID tags or sensor networks, enable logistics objects like products, containers or even ships to interact and autonomously decide on their upcoming actions. These so-called "smart parts" [1] triggered a discussion about the pros and cons of a decentralization of decision-making in logistics processes, e.g. Ref. [2]. The underlying idea is based on a shift from an important degree of external control to a higher degree of the organizational principle of autonomous cooperation [3]. Following this concept consistently, supply networks can then be regarded as complex adaptive logistics systems (CALS) [1,4-7]. Inspired by the exceptional performances obtained by populations of social insects able, via mutual interaction mechanisms, to self-organize and ultimately produce robust and highly efficient patterns (e.g. Ref. [8]), modern management aims to favor decentralized decision processes. Decentralization is implemented by introducing a collection of suitable agents able to process local information in real time. These agents are endowed with an autonomous decision capability and their choices are based on this local and incomplete information (i.e. only a part of the population is usually observed). The multiple interactions between neighboring agents are ultimately expected to generate an emergent global behavior that, due to self-organization, should ideally outperform a centralized decision process.

However, effects of a reduction of external control and an increase of the degree of autonomous cooperation in transportation logistics on performance indicators, such as delivery time or delivery costs, have not been shown yet in analytically solvable models. Inspired by this logistics field of application, the aim of the present paper is to propose a stylized, fully solvable transportation problem for which this paradigmatic view can be mathematically observed. Benefits of self-organization have notably been pointed out in the context of urban road networks. In Ref. [9], a fluid-dynamic model is proposed to unveil that self-control of traffic lights and vehicle flows might lead to efficient traffic processes. As intersecting flows of vehicular traffic are often managed by an external control that might lead to oscillatory behaviors, decentralized control strategies become thus interesting and fruitful alternatives, [10,11]. Also somehow related to the present study, a model of commuters having the choice between alternative roads and basing their routing decisions on their neighbors' most recent waiting experience and on their own complete waiting history (i.e. on their exponentially weighted average experimented waiting times for each road) is proposed in Ref. [12]. This contribution explicitly exhibits the striking feature that a self-organizing system based on local information and locally rational agents might outperform (i.e. the average travel time of commuters is reduced) the Nash equilibrium (that assumes full information).

The complex evolution of statistical mechanics models involving multi-agent features usually precludes any analytical approaches, with the exception of the case when large and intimately connected populations are considered. In this case, the law of large numbers enables one to collect the numerous mutual interactions due to neighboring agents into an effective external field. The resulting mean-field approximate dynamics is described by time-dependent nonlinear field equations for which some analytical information can be extracted. This is precisely the method that we will follow in this contribution and which will lead to explicit solutions.

Stylized models, as the one introduced in this work, handle the complexity by reducing drastically the number of considered parameters. The aim of such kinds of models is to remain analytically tractable but to allow nevertheless for a detailed understanding of the origin and nature of the emerging phenomena. Moreover, the desired simplicity of these models implies that changes and variants can be addressed in a simple way, in order to eventually incorporate additional features. The approach consisting in developing such types of stylized models has been recently adopted in economics [13,14]. In these contributions, a minimal agent-based model for financial markets is developed in order to understand the essence of several collective self-organized patterns that appear in price evolution. Closely related to the present methodology and without being exhaustive, let us also quote stylized multi-agent models in the field of investment behaviors in stock markets [15] and autonomous routing in queueing systems [16].

The paper proceeds as follows: Section 2.1 depicts the basic dynamics of the model under consideration and thus consists in a description of the regarded population of agents, their decision rules and interaction patterns as well as the noise sources affecting the agents' dispersion over time. Taking this as a basis for the analysis that follows, Section 2.2 provides under a mean-field approach (i.e. we consider homogeneous agents) an analytical solution to the considered dynamics, notably with regard to the resulting acceleration effects of the whole cluster of agents. Section 2.3 consists in a cost function analysis, the aim of which is to compare the cumulative costs that result from autonomous agents' interactions with those that would occur if an effective optimal central controller would lead the cluster of non-interacting agents to an equal dispersion. Section 3 is devoted to concluding remarks; the findings are summed up and future and further research requirements are pointed out.

\section{Self-organized transportation based on an idealized smart parts paradigm}

\subsection{Basic dynamics}

Consider a collection of agents $\mathcal{A}_{k}, k=1,2, \ldots, N$, with evolution given by the stochastic dynamics:

$$
\dot{X}_{k}(t)=v_{k}(t)+\gamma_{k} \underbrace{\mathbb{I}_{k}(\vec{X}(t))}_{\text {agent interactions }}+q_{k}\left(\vec{X}(t), v_{k}(t)\right) \mathrm{d} B_{k, t},
$$


where $X_{k}(t)$ stands for the distance traveled by agent $\mathcal{A}_{k}, \vec{X}(t)$ is defined as:

$$
\vec{X}(t)=\left(X_{1}(t), X_{2}(t), \ldots, X_{N}(t)\right),
$$

$v_{k}(t)$ are individual velocities and $\mathbb{I}_{k}(\vec{X}(t))$ is a dimensionless coupling term with velocity strength $\gamma_{k}$ describing interactions between the agents. When $\gamma_{k}=0$ (i.e. no interactions between the agents) we define an ideal distance $W_{k}(t)$ traveled during time $t \mathrm{as}^{2}$ :

$$
W_{k}(t)=\int_{0}^{t} v_{k}(s) \mathrm{d} s .
$$

The terms $q_{k}\left(\vec{X}(t), v_{k}(t)\right) \mathrm{d} B_{k, t}$ stand for the respective noise sources the amplitudes of which may generally depend on the agents' positions and velocities. The term $\gamma_{k} \mathbb{I}_{k}(\vec{X}(t)) \geq 0$ describes how agent interactions affect the agents' individual velocities $\left(\mathbb{I}_{k}(\vec{X}(t))\right.$ is dimensionless and $\gamma_{k}$ has the dimension of a velocity).

Having in mind a logistics context, we may view $X_{k}(t)$ as the position of a "smart container" (i.e. agent $\left.\mathcal{A}_{k}\right)$ traveling with $v_{k}(t)$ as an ideal predefined velocity. The random environment is idealized with the noise source $q_{k}\left(\vec{X}(t), v_{k}(t)\right) \mathrm{d} B_{k, t}$. Numerous sources of fluctuations are conceivable, for example environmental and/or measurement noises can be considered. The "smart" agent character is introduced via the $\mathbb{I}_{k}(\cdot)$ term which we will define as:

$$
\mathbb{I}_{k}(\vec{X}(t))=\frac{1}{\mathcal{N}_{k}} \sum_{j \neq k}^{\mathcal{N}_{k}} \ell_{k}\left(X_{j}(t)\right),
$$

where $\mathcal{N}_{k}$ is the set of "neighboring" agents observable by agent $\mathcal{A}_{k}$ and, for $j \in\left\{1,2, \ldots, k-1, k+1, \ldots, \mathcal{N}_{k}\right\}$ :

$$
\ell_{k}\left(X_{j}(t)\right)= \begin{cases}0 & \text { if } 0 \leq X_{j}(t)<X_{k}(t), \\ 1 & \text { if } X_{k}(t) \leq X_{j}(t)<X_{k}(t)+U,(U>0), \\ 0 & \text { if } X_{j}(t) \geq X_{k}(t)+U .\end{cases}
$$

In other words, agent $\mathcal{A}_{k}$ observes the positions occupied by $\mathcal{N}_{k}$ of its neighbors:

- When $X_{k}(t)>X_{j}(t)$, agent $\mathcal{A}_{k}$ does not modify its velocity ( $\mathcal{A}_{k}$ feels that he is in advance).

- When $X_{k}(t) \leq X_{j}(t)<X_{k}(t)+U$, agent $\mathscr{A}_{k}$ feels that he is late compared with $\mathcal{A}_{j}$, accordingly he increases his velocity to try to match $\mathcal{A}_{j}$ 's behavior.

- When $X_{j}(t) \geq X_{k}(t)+U$, agent $\mathcal{A}_{k}$ feels that he is far too late and he does not attempt to catch $\mathcal{A}_{j}$.

Thanks to the introduction of White Gaussian Noise (WGN) into the dynamics given by Eq. (1), the evolution of the system is described by a $N$-dimensional diffusion process which we may alternatively represent as a $N$-dimensional transition probability density $P(\vec{X}(t), t \mid 0,0):=P(\vec{X}, t)$ solving a Fokker-Planck (parabolic) diffusion equation. This alternate representation becomes straightforward in the case of a population of homogeneous agents $\left(i . e . v_{k}(t) \equiv v(t), W_{k}(t) \equiv W(t)\right.$ and $\gamma_{k} \equiv \gamma$ ) without interaction (i.e. $\left.\ell_{k}\left(X_{j}(t)\right) \equiv 0\right)$ and when $\mathrm{d} B_{k, t}$ are independent White Gaussian Noise (WGN) processes, $\forall k=1,2, \ldots, N$. From now on we will moreover focus on additive noise sources for which we have $q_{k}\left(\vec{X}(t), v_{k}(t)\right) \equiv q$ In this case, we obtain the following $N$-multivariate Gaussian distribution for $P(\vec{X}(t), t \mid 0,0)$ :

$$
P(\vec{X}(t), t \mid 0,0)=\left\{g\left(W(t), \sigma_{q}^{2}(t)\right)\right\}^{N},
$$

where $g\left(m, \sigma^{2}\right)$ denotes a Gaussian density with mean $m$ and variance $\sigma^{2}$.

\subsection{Mean-field approach for homogeneous agents}

To discuss analytically the model given by Eq. (1), we now focus on situations with large number of observable agents $\mathcal{N}_{k} \gg 1, \forall k=1,2, \ldots, N$, and specifically we will consider the cases where $\mathcal{N}_{k}=\chi_{k} N$, with $\left.\left.\chi_{k} \in\right] 0,1\right]$ fixed proportions, and $\mathcal{N}_{k} \rightarrow \infty$ (every agent observes a very large neighboring population). As before, the noise generators $\mathrm{d} B_{k, t}, k=1,2, \ldots, N$ are taken to be independent WGN processes. In the homogeneous case where $\chi_{k} \equiv \chi$ and when $\mathcal{N}_{k} \equiv \mathcal{N} \rightarrow \infty$, the MeanField Dynamics (MFD) approach consists in writing:

$$
P(\vec{X}(t), t \mid 0,0)=\{P(x, t) \mathrm{d} x\}^{N},
$$

where the single effective transition probability density $P(x, t)$ solves the Fokker-Planck equation:

$$
\frac{\partial}{\partial t} P(x, t)=-\frac{\partial}{\partial x}\left[\mathscr{D}_{v}(x, t) P(x, t)\right]+\frac{1}{2} q^{2} \frac{\partial^{2}}{\partial x^{2}}[P(x, t)],
$$

\footnotetext{
2 The functions $v_{k}(t)$ denote velocities, hence the physical dimension of $W_{k}(t)$ is a distance.
} 
where the drift field $\mathscr{D}_{v}(x, t)$ reads as:

$$
\mathscr{D}_{v}(x, t)=v(t)+\gamma \underbrace{\left\{\int_{x(t)}^{x(t)+U} P(x, t) \mathrm{d} x\right\}}_{\varphi_{U}(x, t)},
$$

with $\gamma$ as before having the dimension of a velocity. The dimensionless term $\varphi_{U}(x, t)$ represents the probability density to find, at time $t$, agents at position $x(t)$ satisfying $x(t) \leq x \leq x(t)+U$. The amplitude of $\varphi_{U}(x, t)$ therefore modulates the velocity correction due to the agents' interactions and hence $U$ has to be interpreted as a dimensionless sensitivity factor. For homogeneous agents (i.e. $\mathcal{N}_{k} \equiv \mathcal{N}$ ), the MFD approach consists in writing Eqs. (3) and (4) as:

$$
\frac{1}{\mathcal{N}} \sum_{j \neq k}^{\mathcal{N}} \ell_{k}\left(X_{j}(t)\right) \approx \int_{x(t)}^{x(t)+U} P(x, t) \mathrm{d} x .
$$

Solving the diffusion given by Eq. (7) with the drift given by Eq. (8) offers in general little hope for analytical discussion (one deals with non-linear and non-local field equation). However for small values of the sensitivity factor $U$, we may Taylor expand Eq. (8) and, to first order in $U$, Eq. (7) can be rewritten as:

$$
\frac{\partial}{\partial t} P(x, t)=-\frac{\partial}{\partial x}\left[v(t) P(x, t)+\gamma U P^{2}(x, t)\right]+\frac{1}{2} q^{2} \frac{\partial^{2}}{\partial x^{2}}[P(x, t)] .
$$

Introducing the change of variables

$$
x \mapsto y=x-W(t),
$$

the systematic deterministic velocity drift $v(t)$ will be removed from Eq. (10) and we obtain:

$$
\frac{\partial}{\partial t} P(y, t)=-\gamma U \frac{\partial}{\partial y}[P(y, t)]^{2}+\frac{q^{2}}{2} \frac{\partial^{2}}{\partial y^{2}}[P(y, t)],
$$

which is the famous viscous Burgers' equation. We have to complete Eq. (12) with an initial condition which we will take here as:

$$
P(y, t=0)=\delta(y), \quad(\delta \text { is the Dirac measure }) .
$$

By introducing the rescaling

$$
t \mapsto \tau=\gamma t, \quad y \mapsto z=\frac{y}{2 U},
$$

we transform Eq. (12) into the dimensionless Burgers' equation:

$$
\frac{\partial}{\partial t} P(z, t)=-\frac{1}{2} \frac{\partial}{\partial z}[P(z, t)]^{2}+\left[\frac{q^{2}}{8 U^{2} \gamma}\right] \frac{\partial^{2}}{\partial z^{2}}[P(z, t)] .
$$

It is well known that Eq. (15) can be linearized into the heat equation via a logarithmic transformation discovered by Hopf and Cole, [17]. This transformation reads as:

$$
P(z, t)=-\frac{\partial}{\partial z} \ln (\Phi(z, t)) \Rightarrow \dot{\Phi}(z, t)=\left[\frac{q^{2}}{8 U^{2} \gamma}\right] \frac{\partial^{2}}{\partial z^{2}} \Phi(z, t) .
$$

Using Eq. (16) and for the initial condition given by Eq. (13), the solution of Eq. (15) is easy to derive and is given in Ref. [17]. According to the change of variables given in Eq. (14), the normalized solution of Eq. (12) hence reads as:

$$
\begin{aligned}
P(y, t) & =-\frac{q^{2}}{4 \gamma U^{2}} \frac{\partial}{\partial y} \ln \left[1+\frac{\left(e^{R}-1\right)}{2} \operatorname{Erfc}\left(\frac{y}{q \sqrt{t}}\right)\right] \\
& =\frac{1}{R}\left[\frac{\frac{\left(e^{R}-1\right)}{\sqrt{\pi q^{2} t}} e^{-\frac{y^{2}}{q^{2} t}}}{1+\frac{\left(e^{R}-1\right)}{2} \operatorname{Erfc}\left(\frac{y}{q \sqrt{t}}\right)}\right] \\
& =: \frac{1}{R} \frac{\left(e^{R}-1\right) \mathbb{G}(y, t)}{\mathbb{E}(y, t)},
\end{aligned}
$$

where $\mathbb{G}(y, t)$ and $\mathbb{E}(y, t)$ are defined as:

$$
\mathbb{G}(y, t)=\frac{1}{\sqrt{\pi q^{2} t}} e^{-\frac{y^{2}}{q^{2} t}}, \quad \mathbb{E}(y, t)=1+\frac{\left(e^{R}-1\right)}{2} \operatorname{Erfc}\left(\frac{y}{q \sqrt{t}}\right)
$$




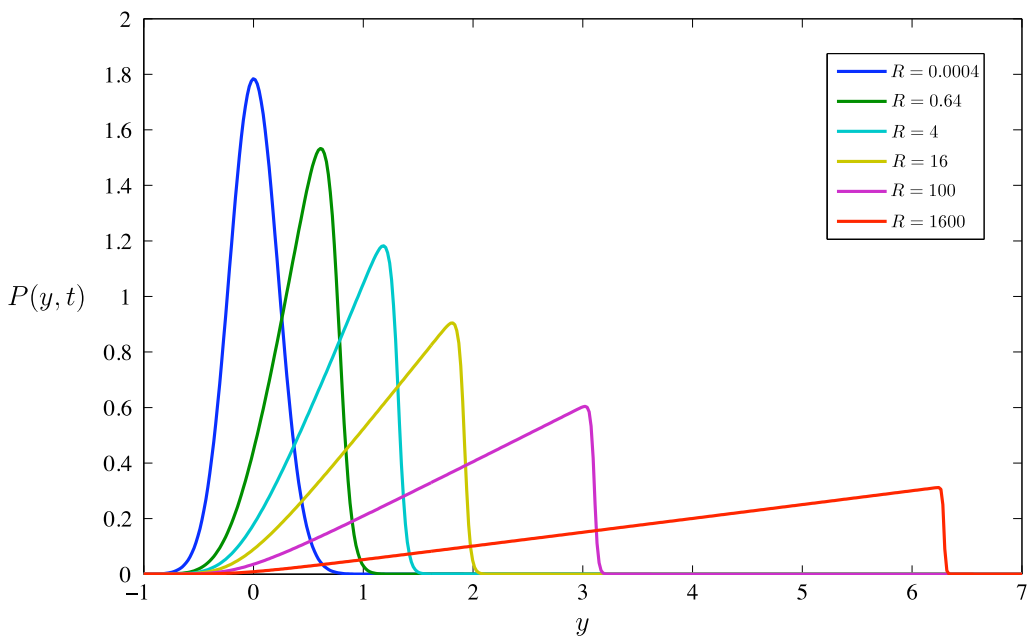

Fig. 1. Probability density function $P(y, t)$ characterizing the spatial dispersion of the agents at time $t=10$ for $N=2000$ and $q=0.1$. This graph is a representation of the solution of the presently considered Burgers' equation, given by Eq. (17), for different values of the control parameter $R$. The two limiting regimes are clearly observable in blue $(R \rightarrow 0)$ and in red $(R \rightarrow \infty)$. Note that the solutions have been translated around zero $(i . e$. the point $y=0$ in the graph corresponds actually to the point $y=\int_{0}^{t=10} v(s) \mathrm{d} s$ of the dynamics). (For interpretation of the references to colour in this figure legend, the reader is referred to the web version of this article.)

and where the dimensionless parameter $R$ is defined as:

$$
R:=\frac{4 U^{2} \gamma}{q^{2}}
$$

Two limiting regimes can be identified:

(a) Strongly diffusive regime obtained for large fluctuation amplitudes, i.e. when $R \rightarrow 0$ (in this case, the random perturbations are predominant compared to the self-organized process involved by the agents' interactions). In this limiting regime, we approximately have:

$$
P(y, t) \simeq \frac{1}{\sqrt{\pi q^{2}} t} e^{-\frac{y^{2}}{q^{2} t}} .
$$

(b) Strongly interactive regime obtained for vanishing fluctuation amplitudes, i.e. when $R \rightarrow \infty$ (in this case, the selforganized mechanism involved by the agents' interactions predominates over the random fluctuations). In this limiting regime, we obtain:

$$
P(y, t) \simeq \frac{y}{4 U^{2} \gamma t}, \quad \text { for } 0 \leq y \leq \sqrt{8 U^{2} \gamma t} .
$$

The moments of the density given by Eq. (20) read as:

$$
\left\langle Y^{n}(t)\right\rangle=\int_{0}^{\sqrt{8 U^{2} \gamma t}} \frac{y^{n+1} \mathrm{~d} y}{4 U^{2} \gamma t}=\frac{2^{n+1} U^{n}}{n+2}(2 \gamma t)^{n / 2} .
$$

Hence in this limiting regime, the increase of the average traveled distance due to agents' interactions is obtained by taking $n=1$ in Eq. (21), yielding $\langle Y(t)\rangle=\frac{4 U \sqrt{\gamma t}}{3}$.

Comparing the two limiting regimes given by Eqs. (19) and (20), it is interesting to observe that $P(y, t)$ has unbounded support when $R \rightarrow 0$ but bounded support when $R \rightarrow \infty$ (shock wave behavior).

As intuitively expected, when $\gamma R>0$, agents' interactions enhance the velocity of the entire population of agents. Numerical computations of the first moment of the density given by Eq. (17) are reported in Table 1 where the global velocity increase of the whole cluster of agents can be quantitatively appreciated. The solutions given by Eq. (17) of Burgers' equation, for different values of $R$, are illustrated in Fig. 1 and the dynamics of the spatial dispersion of the agents over time is sketched in Fig. 2.

\subsection{Cost function analysis}

Having observed the acceleration effects that are due to autonomous agents' cooperation, we may now infer on its efficiency. To this aim, we introduce a class of effective centralized controllers yielding, for a given time horizon $T$, a similar agent probability distribution. 


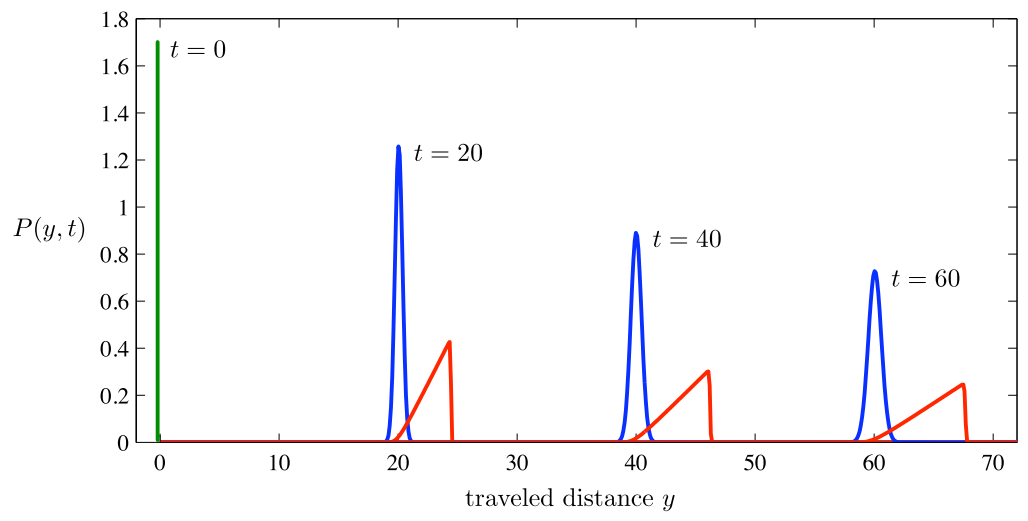

Fig. 2. Dynamics of the agents' spatial dispersion for $N=2000, q=0.1, \gamma=1$ and $v(t)=1, \forall t>0$. A pool of entities are traveling from point $\mathbf{A}=0$ to point $\mathbf{B}=70$. Initially (i.e. at $t=0$ ), all the items are located at $y(t=0)=y_{0}=0_{-}$(which is represented by the green delta-peak function on the graph above). At time $t=20$, due to the noise affecting their evolution, the entities are dispersed around the originally planned trajectory $y(t=20)=\int_{0}^{20} v(s) \mathrm{d} s=20$. Without almost any control on the dynamics of the entities $\left(R=1.6 \cdot 10^{-7}\right.$, i.e. practically no interactions between the agents), the dispersion follows a Gaussian distribution with mean $y(t=20)=20$ (in blue on the graph). When agents are strongly interacting $(R=100)$, the dispersion is deeply modified by the agents' autonomous local interactions and follows the density drawn in red above. At $t=40$, the originally planned schedule is obviously found to be even closer to $\mathbf{B}$, at $y(t=40)=\int_{0}^{40} v(s) \mathrm{d} s=40$, and the densities describing the dispersion of the agents are, due to diffusion, more flat compared with $t=20$. However, one might observe in this graph that the Kullback-Leibler distance between the blue and red lines remains constant at any time of the dynamics. (For interpretation of the references to colour in this figure legend, the reader is referred to the web version of this article.)

Table 1

Average traveling enhancement $\langle y(t)\rangle$ of the whole population of agents. The values presented below correspond to the probability density functions of Fig. 1, again with fixed time $t=10$.

\begin{tabular}{lll}
\hline & $R$ & $\langle y(t)\rangle=\int_{-\infty}^{+\infty} y P(y, t) \mathrm{d} y$ \\
\hline 0 & 0 & 0 \\
& 0.2 & 0.012 \\
& 2 & 0.126 \\
20 & 0.824 \\
40 & 1.219 \\
& 100 & 2.023 \\
& 200 & 2.914 \\
\hline
\end{tabular}

For the MFD regime, we have obtained the multivariate probability density $P(\vec{x}, t) \mathrm{d} x_{1} \mathrm{~d} x_{2} \cdots \mathrm{d} x_{N}=[P(x, t) \mathrm{d} x]^{N}$ with $P(x, t)$ solving, up to the translation given by Eq. (11), the Burgers' equation. The same probabilistic behavior can also be obtained by introducing an effective centralized controller which takes care of each agent by simultaneously implementing a set of identical drifts $c_{k}\left(X_{k}, t\right) \equiv c\left(X_{k}, t\right)$ controlling each individual agent. We will focus on a class of effective centralized controllers that only depend on the final state to be reached by the population of agents at a given time horizon $T$. Despite to the fact that our class of effective controllers involves state- and time-dependent drifts, it has to be clearly distinguished from the class of real time information controllers which permanently re-actualize their states based on updated local information sets (here only the initial and the final states to be reached are taken into account). To construct our effective centralized controller, one considers $N$ independent diffusion processes of the type:

$$
\mathrm{d} X_{k, t}=c_{k}\left(X_{k}, t\right) \mathrm{d} t+\frac{q}{2} \mathrm{~d} B_{k, t}, \quad X_{k, 0}=0, \quad k=1,2, \ldots, N,
$$

yielding a set of probability densities $P_{c_{k}}(x, t) \equiv P_{c}(x, t)$, for $k=1,2, \ldots, N$. For a given time horizon $T>0$, we will impose that:

$$
\left[P_{c}(x, T) \mathrm{d} x\right]^{N}=[P(x, T) \mathrm{d} x]^{N},
$$

i.e. the dynamics obtained for centralized and decentralized controls lead to the same final dispersion of agents. However, it is important to note here that, while the final probability measures (i.e. at time $T$ ) coincide for both type of controls, the dynamics differs during the time interval ]0,T[. Such distinct transient evolutions give rise, as we will observe in Section 2.3.3, to different global cumulative costs.

Let us now define and compare the costs incurred under the original decentralized multi-agent evolution given by Eq. (1) with those resulting from the implementation of an optimal effective centralized controller with $\operatorname{drift} c_{k}^{*}\left(X_{k}, t\right)$ and for which Eq. (23) is satisfied. We will consider a cost rate function based on the ratio between the kinetic energy due to the velocity 
adjustments and the diffusion rate due to noise. Hence, roughly speaking, our cost rate function can also be understood as a signal-to-noise ratio.

\subsubsection{Costs incurred by decentralized velocity adjustments}

Let us assume that a cost is only incurred when an agent is speeding up according to the rule given by Eq. (4). For a collection of $N$ agents and for a time horizon $T$, the global (cumulative) costs $J_{\text {agents }, T}$ can formally be written as:

$$
J_{\text {agents, } T}=\sum_{k=1}^{N} \int_{0}^{T} \frac{1}{q^{2}} \underbrace{\frac{\gamma^{2}}{2}\left[\frac{1}{\mathcal{N}} \sum_{j \neq k}^{\mathcal{N}} \ell_{k}\left(X_{j}(t)\right)\right]^{2}}_{\text {kinetic energy of agent, } \mathcal{A}_{k}} \mathrm{~d} t \leq N \frac{\gamma^{2} U^{2}}{2 q^{2}} T,
$$

where according to Eq. (4), the term $\frac{1}{\mathcal{N}} \sum_{j \neq k}^{\mathcal{N}} \ell_{k}\left(X_{j}(t)\right) \in[0,1]$ stands for the proportion of agents among the whole population currently interacting with agent $\mathcal{A}_{k}$ at time $t$.

\subsubsection{Costs of an equivalent effective central controller}

Let us now examine the possibility to reach the probability density state given by Eq. (17) by using, instead of interactive agents, an effective central controller of the form introduced in Eq. (22). Remember that costs are incurred here only for velocity increase. We now focus on a diffusion process given by:

$$
\mathrm{d} Y_{t}=c(Y, t) \mathrm{d} t+\frac{q}{2} \mathrm{~d} B_{t}, \quad Y_{0}=0, \quad(0 \leq t \leq T),
$$

where $c(Y, t)$ is a drift controller. The dynamics given by Eq. (25) leads to the following Fokker-Planck equation:

$$
\frac{\partial}{\partial t} P_{c}(y, t)=-\frac{\partial}{\partial y}\left[c(y, t) P_{c}(y, t)\right]+\frac{q^{2}}{2} \frac{\partial^{2}}{\partial y^{2}} P_{c}(y, t) .
$$

Given a time horizon $T>0$, we would like now to construct a controller $c(Y, t ; T)$ which ultimately produces (i.e. at time $T$ ) the same final effect as the spontaneous agents' interaction mechanism. In other words and in view of Eq. (23), we would like to find $c(Y, t ; T)$ such that the solution:

$$
\underbrace{P_{c}(y, T)}_{\text {solution of Eq. (26) }}=\underbrace{P(y, T)}_{\text {density given by Eq. (17) }}
$$

In full analogy with Eq. (24), we can associate a cost function $J_{\text {central, } T}[c(y, t ; T)]$, representing the global (cumulative) costs incurred by the effective centralized control, defined as:

$$
J_{\text {central }, T}[c(y, t ; T)]=\left\langle\left\{\int_{0}^{T} \frac{c^{2}(y, s ; T)}{2 q^{2}} \mathrm{~d} s\right\}\right\rangle,
$$

where $\langle\cdot\rangle$ stands for the averaging over the realizations of the underlying stochastic process. Now we consider the optimal control problem consisting in the construction of the optimal controller $c^{*}(y, t ; T)$ which simultaneously fulfills Eq. (27) and minimizes the cost function given by Eq. (28), namely:

$$
J_{\text {central }, T}\left[c^{*}(y, t ; T)\right] \leq J_{\text {central }, T}[c(y, t ; T)]
$$

over all possible controls $c(y, t ; T)$.

The explicit solution of this stochastic optimal control problem has been given by Dai Pra in Ref. [18] and can be summarized as follows:

(a) Optimal controller $c^{*}(y, t ; T)$. The optimal effective controller is given by:

$$
c^{*}(y, t ; T)=\frac{\partial}{\partial y} \ln [h(y, t)],
$$

with

$$
h(y, t)=\int_{\mathbb{R}} \mathbb{G}[(z-y),(T-t)] \frac{P(z, T)}{\mathbb{G}(z, t)} \mathrm{d} z
$$

and where the functions $P(y, t)$ and $\mathbb{G}(y, t)$ are defined in Eqs. (17) and (18).

(b) Minimal cost value. The optimal minimum cumulative costs $J_{\text {central }, T}\left[c^{*}(y, t ; T)\right]=\mathscr{D}(P \mid \mathbb{G})$ where $D(P \mid \mathbb{G})$ is the Kullback-Leibler relative entropy (or pseudo-distance) between the free evolution (here given by $\mathbb{G}(y, t)$ ) and the controlled density $P(y, t)$ given by Eq. (17). 
Remember that for two probability densities $p(y)$ and $q(y)$, the Kullback-Leibler relative entropy $\mathscr{D}(p \mid q)$ reads as:

$$
\mathscr{D}(p \mid q)=\int_{\mathbb{R}} p(y) \ln \left[\frac{p(y)}{q(y)}\right] \mathrm{d} y .
$$

Here, we have $p(y)=P(y, t)$ given by Eq. (17) and $q(y)$ is the normalized Gaussian density $q(y, t)=\mathbb{G}(y, t)=\frac{1}{\sqrt{\pi q^{2} t}} e^{-\frac{y^{2}}{q^{2} t}}$. Using the properties:

$$
\begin{aligned}
& \int_{\mathbb{R}} \mathbb{G}(y, t) \mathrm{d} y=1, \quad \int_{\mathbb{R}} P(y, t) \mathrm{d} y=1, \quad \frac{\partial \mathbb{E}(y, t)}{\partial y}=\left(e^{R}-1\right) \mathbb{G}(y, t), \\
& \mathbb{E}(\infty)=1 \text { and } \mathbb{E}(-\infty)=e^{R},
\end{aligned}
$$

we can write:

$$
\begin{aligned}
\mathscr{D}(p \mid q) & =\int_{\mathbb{R}} \frac{\left(e^{R}-1\right) \mathbb{G}}{R \mathbb{E}} \ln \left[\frac{\left(e^{R}-1\right) \mathbb{G}}{R \mathbb{E} \mathbb{G}}\right] \mathrm{d} y \\
& =\left.\frac{1}{R} \ln \left(\frac{e^{R}-1}{R}\right) \ln (\mathbb{E})\right|_{-\infty} ^{\infty}-\int_{\mathbb{R}} \frac{\left(e^{R}-1\right) \mathbb{G}}{R \mathbb{E}} \ln (\mathbb{E}) \mathrm{d} y \\
& =\ln \frac{\left(e^{R}-1\right)}{R}-\frac{1}{R} \int_{\mathbb{R}}\left(\frac{\mathrm{d}}{\mathrm{d} y} \ln (\mathbb{E})\right) \ln (\mathbb{E}) \mathrm{d} y \\
& =\ln \frac{\left(e^{R}-1\right)}{R}-\left.\frac{1}{2 R}[\ln (\mathbb{E})]^{2}\right|_{-\infty} ^{\infty} \\
& =\ln \frac{\left(e^{R}-1\right)}{R}+\frac{R}{2} .
\end{aligned}
$$

Hence, for a population of $N$ agents, we end with a global (cumulative) costs value equal to:

$$
J_{\text {central }, T}\left[c^{*}(y, t ; T)\right]= \begin{cases}N D(P \mid \mathbb{G})=\frac{N R}{2}+N \ln \left[\frac{\left(e^{R}-1\right)}{R}\right] & \text { for } T>0, \\ 0 & \text { for } T=0 .\end{cases}
$$

For time horizon $T=0$, the result follows directly as, for time $t=0$, we have assumed that $P(y, 0)=\mathbb{G}(y, 0)=\delta\left(y=0_{-}\right)$ (see Ref. [19]). One might observe in Eq. (32) the truly remarkable feature that $\mathscr{D}(P \mid \mathbb{G})$ is, for $T>0$, only dependent on $R$ (and not on the time horizon $T$ ). As the solutions of the Burgers' equation and the heat equation are, for all times, connected via the Hopf-Cole transformation given by Eq. (16), their relative entropy is a constant of the motion. For strongly diffusive regimes, we have $\lim _{R \rightarrow 0} \mathscr{D}(P \mid \mathbb{G})=0$ as it should be since agent interactions are nearly negligible in this limit and hence no costs are incurred. Conversely for strongly interacting regimes, we have $\lim _{R \rightarrow \infty} \mathscr{D}(P \mid \mathbb{G}) \simeq R / 2$.

Observe that while the global cumulative costs given by Eq. (32) remain constant and independent on the given time horizon $T$, the effective optimal drift itself, as given by Eq. (30), strongly depends on $T$. Hence, defining an optimal average cost rate $r_{\text {central }, T}^{*}:=\frac{1}{T} J_{\text {central }, T}\left[c^{*}(y, t ; T)\right]$, we then conclude that the larger is $T$, the smaller will be $r_{\text {central }, T}^{*}$.

\subsubsection{Costs comparison}

Let us now compare the global cumulative costs $J_{\text {agents, },}$ and $J_{\text {central }, T}\left[c^{*}(y, t ; T)\right]$. Invoking Eqs. (24) and (32) and due to the fact that the multi-agent cumulative costs are monotonously increasing with $T$, we can conclude that it exists a critical time horizon $T_{c}$ such that:

$$
J_{\text {agents }, T}<J_{\text {central }, T}\left[c^{*}(y, t ; T)\right], \quad \text { for } T<T_{c} .
$$

Hence, in this stylized modeling framework, we are able to explicitly observe that for time horizons shorter than $T_{c}$, the agent-based self-organization beats the optimal effective centralized controller. This striking feature can actually be intuitively understood. For small horizons $T<T_{c}$, all agents are confined in a relatively compact cluster and therefore, according to the interaction rule given by Eq. (4), almost all agents accelerate. This almost global change of velocities generates, for small time horizons, a very high cost rate $r_{\text {central.T }}^{*}$ for the effective central control. This phenomenon favors the self-organized behavior when a short time horizon $T$ is considered. Alternatively, for larger values of $T$, the noise-induced diffusive behavior strongly reduces the effective strength of the interactions, thus decreasing $r_{\text {central }, T}^{*}$. In our model, this cost reduction for time horizons $T>T_{c}$ favors the optimal effective centralized controller. This picture is sketched in Fig. 3. Note that, for the goal of the present contribution, the upper-bound for the decentralized costs (see Section 2.3.1) is sufficient to establish the existence of an interval $\left[0, T_{c}\right]$ of time horizons for which decentralization is advantageous and should hence be favored. 


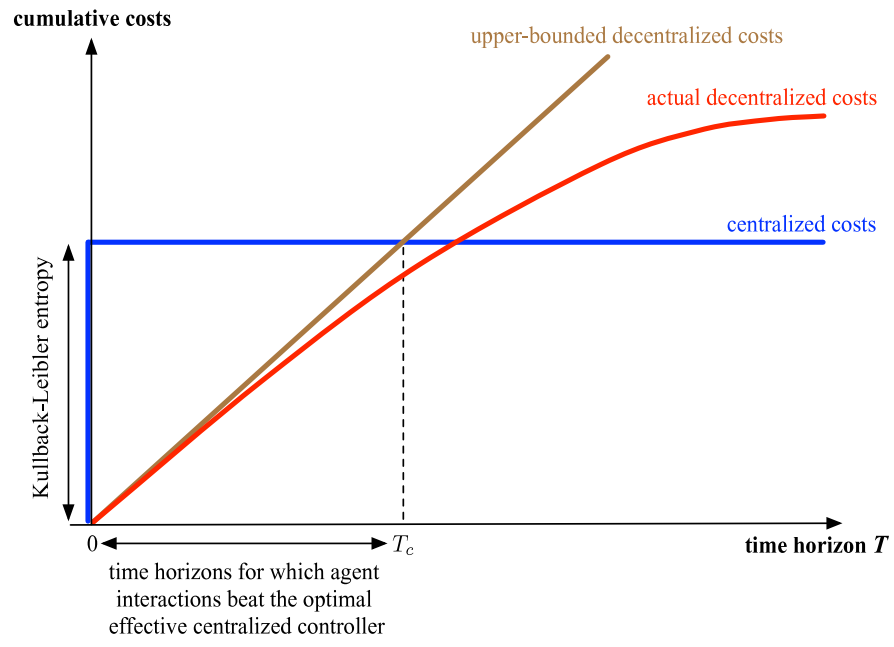

Fig. 3. Qualitative sketch of the global cumulative costs incurred by decentralized and effective centralized control.

\section{Conclusion}

The aim of this paper was to present a stylized, fully solvable model that represents a logistics transportation scenario in which decision-making has been decentralized and shifted to single logistics objects that are able to interact with the help of modern information and communication technologies, such as RFID tags or sensor networks. It is rather widely accepted that the management and control of highly complex production systems and/or supply chains networks does, at least partly, rely on such decentralized mechanisms of the multi-agent type. For given utility functions, the hope is that from the agents' interactions will ultimately emerge adaptive, self-organized and hopefully efficient management policies.

Complex adaptive logistics systems, realized through the application of technologies based on autonomous cooperation, exhibit exactly these characteristics and provide therefore potentialities to enhance the performances of the underlying supply networks [7]. However, as is the rule for most actual situations, it is very likely that the optimal management rules will be an optimal mix of centralized and decentralized policies. This hence raises a somehow philosophically inspired question, namely to determine the right compromise between interventionism and autonomy? Our present model, though highly stylized, exhibits quantitatively the somehow intuitive feeling that if, in a short time slot, a huge number of interactions occurs, a decentralized control of the multi-agent type outperforms an effective central management. We hope that our present model contributes to transform what perhaps could, at first sight, be ranked as a hollow wish into a more palpable management possibility.

Therefore, future research is required that deals with an increase of the complexity, such that the model is able to represent and to achieve less general but instead more specific results. In analogy to these future requirements and specific research on complex adaptive logistics systems, advances would result from the need for a further specification and adaptation of the underlying agents' interaction and decision mechanisms as well as the applied cost functions, in order to implement the performance improvements shown here in real logistics scenarios.

\section{Acknowledgements}

The authors are very grateful to the referees who reviewed the present paper and whose comments have enabled to significantly improve the presentation of its content. MOH and OG would like to thank Dr. R. Filliger for several enlightening discussions and comments. This research has been supported by the Swiss National Science Foundation (FNS) under grant No. 200020-117608 and by the German Research Foundation (DFG) as part of the Collaborative Research Centre 637 "Autonomous Co-operating Logistic Processes - A Paradigm Shift and its Limitations".

\section{References}

[1] T.Y. Choi, K.J. Dooley, M. Rungtusanatham, Supply networks and complex adaptive systems: control versus emergence, Journal of Operations Management 19 (3) (2001) 351-366.

[2] M. Hülsmann, J. Grapp, Y. Li, Strategic adaptivity in global supply chains-competitive advantage by autonomous cooperation, International Journal of Production Economics 114 (1) (2008) 14-26.

[3] K. Windt, M. Hülsmann, Changing paradigms in logistics-understanding the shift from conventional control to autonomous cooperation and control, in: M. Hülsmann, K. Windt (Eds.), Understanding Autonomous Cooperation \& Control-The Impact of Autonomy on Management, Information, Communication and Material Flow, Springer, Berlin, 2007, pp. 1-16.

[4] B. McKelvey, C. Wycisk, M. Hülsmann, Designing an electronic auction market for complex 'smart parts' logistics: options based on Lebaron's computational stock market, International Journal of Production Economics 120 (2) (2009) 476-494. 
[5] S.D. Pathak, J.M. Day, A. Nair, W.J. Sawaya, M.M. Kristal, Complexity and adaptivity in supply networks: building supply network theory using a complex adaptive systems perspective, Decision Sciences 38 (4) (2007) 547-580.

[6] A. Surana, S.R.T. Kumara, M. Greaves, U.N. Raghavan, Supply-chain networks: a complex adaptive systems perspective, International Journal of Production Research 43 (20) (2005) 4235-4265.

[7] C. Wycisk, B. McKelvey, M. Hülsmann, Smart parts supply networks as complex adaptive systems: analysis and implications, International Journal of Physical Distribution \& Logistics Management 38 (2) (2008) 108-125.

[8] E. Bonabeau, M. Dorigo, G. Theraulaz, Swarm Intelligence: From Natural to Artificial Systems, Oxford University Press, 1999.

[9] S. Lämmer, D. Helbing, Self-control of traffic lights and vehicle flows in urban road networks, Journal of Statistical Mechanics: Theory and Experiment 4 (2008), art. no. P04019.

[10] D. Helbing, J. Siegmeier, S. Lämmer, Self-organized network flows, Networks and Heterogeneous Media 2 (2) (2007) 198-210.

[11] R. Donner, Multivariate analysis of spatially heterogeneous phase synchronisation in complex systems: application to self-organised control of material flows in networks, The European Physical Journal B 63 (3) (2008) 349-361.

[12] A. van Ackere, E.R. Larsen, Self-organising behaviour in the presence of negative externalities: a conceptual model of commuter choice, European Journal of Operational Research 157 (2004) 501-513.

[13] V. Alfi, M. Cristelli, L. Pietronero, A. Zaccaria, Minimal agent based model for financial markets I: origin and self-organization of stylized facts, The European Physical Journal B 67 (2009) 385-397.

[14] V. Alfi, M. Cristelli, L. Pietronero, A. Zaccaria, Minimal agent based model for financial markets II: statistical properties of the linear and multiplicative dynamics, The European Physical Journal B 67 (2009) 399-417.

[15] A. Corcos, J.-P. Eckmann, A. Malaspinas, Y. Malavergne, D. Sornette, Imitation and contrarian behavior: hyperbolic bubbles, crashes and chaos, Quantitative Finance 2 (2002) 264-281.

[16] O. Gallay, M.-O. Hongler, Circulation of autonomous agents in production and service networks, International Journal of Production Economics 120 (2009) 378-388.

[17] L. Debnath, Nonlinear Partial Differential Equations for Scientists and Engineers, second ed., Birkhäuser, 2005.

[18] P. Dai Pra, A stochastic control approach to reciprocal diffusion processes, Applied Mathematics and Optimization 23 (1991) 313-329.

[19] J.D. Logan, An Introduction to Nonlinear Partial Differential Equations, Wiley, 1994. 